\title{
Rule-Based Dynamic Modification of Workflows in a Medical Domain
}

\author{
R. Müller, E. Rahm \\ Institut für Informatik, Universität Leipzig, Augustusplatz 10/11, 04109 Leipzig \\ $\{$ mueller,rahm\}@informatik.uni-leipzig.de
}

\begin{abstract}
A major limitation of current workflow systems is their lack of supporting dynamic workflow modifications. However, this functionality is a major requirement for next-generation systems in order to provide sufficient flexibility to cope with unexpected situations and failures. For example, our experience with data intensive medical domains such as cancer therapy shows that the large number of medical exceptions is hard to manage for domain experts. We therefore have developed a rulebased approach for partially automated management of semantic exceptions during workflow instance execution. When an exception occurs, we automatically determine which running workflow instances w.r.t. which workflow regions are affected, and adjust the control flow. Rules are being used to detect semantic exceptions and to decide which activities have to be dropped or added. For dynamic modification of an affected workflow instance, we provide two algorithms ( $d r c d$-and $p$-algorithm) which locate appropriate deletion or insertion points and carry out the dynamic change of control flow.
\end{abstract}

\section{Introduction}

The need for dynamic workflow changes to manage new situations and unexpected difficulties during the execution of workflow instances has already been identified as one of the central workflow research issues (e.g. [She97, SK98]). However, most of the proposed approaches assume that a human expert decides which changes have to be applied. Furthermore, they concentrate on transformation frameworks for dynamic structure changes to achieve consistency of the modified workflow instance w.r.t. control and data flow aspects. In contrast to this, it has not yet been investigated sufficiently whether machine agents can be enabled to automatically decide first when a running workflow instance is no longer adequate, and second which changes of the original workflow definition have to be applied to better reflect the new situation.

To address this problem we describe a rule-based approach for the detection of semantic exceptions and for dynamic workflow modification with a focus on medical workflow scenarios. Dynamic workflow changes are triggered by semantic exceptions which, in our domain, are medical events such as a newly detected allergy or a critical laboratory value requiring a change in an ongoing treatment plan (workflow instance). This is done in several steps: First, a rule-based system decides whether a medical event for a patient implies that currently planned or processed medical activities are not appropriate anymore, and whether additional activities become necessary. Second, it is derived which running workflow instances are affected. Third, for an affected 
workflow instance, the relevant workflow modification region is determined. This region depends on the type of an exception $E$ and the "temporal influence" of $E$, so that changes are only applied to relevant areas. Fourth, within the relevant modification region, workflow modifications are processed to adjust the instance w.r.t. the new situation. Fifth, the modified workflow instance is continued.

Our primary focus thus is on (partially) automated dynamic control flow modifications of workflow instances. We concentrate on local modifications such as the automated deletion, replacement and addition of single workflow activity nodes, while the deletion or construction of complete subgraphs is not yet addressed. Data flow aspects are omitted as well, as they can be viewed, from the semantic point of view, as a more technical consequence of control flow changes. Furthermore, due to space restrictions we do not deal with fatal semantic exceptions like the death of a patient which form - from the technical point of view - a relatively trivial type of semantic exceptions (they definitely terminate workflow instances).

The work described here is part of the workflow system HeMATOWORK [MHL+98, MH98], which supports long-term therapy in the domain of distributed cancer therapy and is currently under development at the University of Leipzig. This domain is suitable for workflow management, as the treatment is mainly based on standardized treatment plans. However, as patients react very different to the aggressive long-term treatment, a significant number of treatments has to be adjusted partially and in a patient-specific manner. By automatically adjusting workflow instances we expect our approach to support physicians because the need for timeconsuming manual interactions with the workflow system is reduced. Moreover, the probability to oversee an important semantic exception can be reduced as well. Certainly, automated workflow modification will be possible only up to a certain degree as events and their implications may become arbitrarily complex. But even if the entire implications of an event cannot completely be derived by a machine agent, a partially automated management is already helpful. In particular, it is already helpful to inform the user which workflow instances w.r.t. which control flow areas are affected by a semantic exception, even if the modifications themselves have to be determined manually. Furthermore, the approach is orthogonal to the question at which step during automated modification the decision should be confirmed by a human expert. For example, the insertion of additional drugs certainly should be confirmed by a physician, while additional diagnostic examinations may be inserted without confirmation. While we focus on medical applications, our approach principally is applicable to other domains as well. In particular, the basic algorithms and agents do not make assumptions about the domain-specific structure of events and exceptions.

The paper is organized as follows. Section 2 discusses related work in the context of dynamic workflows. Section 3 describes the subset of Frame Logic and Transaction Logic [KLW95, BK94] used for the representation of events, activities and rules. We use a logic-based approach as the automation of the dynamic modification process requires formal semantics. F-logic has been selected because it provides objectoriented modeling capabilities combined with deductive aspects. Transaction Logic is needed for formal description of database updates. In Section 4 our basic notations of medical events and activities are formalized. Section 5 contains the model of workflow instance execution and event-caused instance interruptions. Section 6 then 
describes the automated workflow modification system with the $d r c d$-and $p$ algorithms. These two algorithms are responsible for locating appropriate deletion or insertion points and for carrying out the dynamic change. Section 7 completes the paper with a discussion of the strengths and limitations of the approach, implementation aspects and future work to be done.

\section{Related Work}

Although a lot of application domains require increased workflow flexibility, this functionality currently is not provided by most commercial workflow systems. Therefore, the development of dynamic workflow systems has been addressed by several researchers during the last years. An overview and classification of dynamic change approaches can be found, for example, in [She97, SK98].

In [RD98], Reichert and Dadam suggest an elaborated workflow execution model and workflow transformation calculus $\left(\mathrm{ADEPT}_{\mathrm{FLEX}}\right)$ which is able to modify the control and data flow at run-time while maintaining correctness and consistency. Related to this, the WASA approach [VW98, Wes98] provides a set of operators which are able to change the control flow w.r.t. activity instances, such as SkipInstance( $i)$ or RepeatInstance $(i)$. However, mechanisms that could enable an automated agent to decide when and what structural change should be applied to a workflow instance are not discussed in both cases.

In the MOBILE system [BJ96, HSS96], (sub-)workflow definitions can be left incomplete at pre-defined points, if a final decision about an appropriate subworkflow logic can only be determined at run-time. An incomplete definition is described in terms of goals and partially defined process patterns. However, methods to automatically derive the workflow completion, when the control flow arrives at this point and relevant data are available, are not discussed by the authors.

Within the WIDE workflow model and architecture, Casati et al. [CGP+97] describe a typology of workflow exceptions and an exception handling approach which is mainly based on condition-reaction pairs; if a condition is violated and an exception raised, an informative (informing an agent) or a corrective reaction is triggered. The latter for instance may be of type CANCEL_TASK or CANCEL_CASE, or, in general, by aborting or redirecting the control flow. However, the authors do not discuss any reaction sequences which are able to modify the running instance through structural changes.

In [MH98], we sketch an approach to modify workflow instances in a knowledgebased manner. Unfortunately, this approach requires meta information within the workflow definitions indicating at which nodes dynamic modification have to be processed. Furthermore, the approach basically is restricted to dynamic workflow hierarchical refinement, and does not support dynamic changes in general.

In summary, to our knowledge, strategies to automatically identify semantic exceptions and change a workflow instance have not yet been investigated sufficiently. 


\section{Representation Methods}

For the purposes of this paper, we use a combination of F-Logic and Transaction Logic (TL) [KLW95, BK94, BK95] to formalize the interaction between semantic exception events, rules and workflow instances. F-Logic is used to represent both the data model for medical events and activities, and rules expressing dependencies between events and activities in a natural object-oriented manner. As F-Logic is a pure retrieval-oriented language and does not support database updates, we additionally use several constructs of $T L$ to express, for example, the generation of new medical orders when semantic exceptions occur ${ }^{1}$.

F-Logic class definitions are of the form:

person [name $=>$ string, birthday $=>$ date], patient::person, physician::person, patient [diagnosis $=>$ string, responsible-doctor $=>$ physician],

physician [degree $=>$ \{trainee, assistant, senior $\}$, patients $=>>$ patient]

where "=>> arcs denote set-valued attributes, and "::" is the $i s-a$ operator. Object instances are expressed, for example, via:

$$
\begin{aligned}
& \text { Bob:patient [name } \rightarrow \text { „Miller, Bob“, birthday } \rightarrow 12 / 5 / 1967, \\
& \text { diagnosis } \rightarrow \text {,Leukemia“, responsible-doctor } \rightarrow \text { Steve] } \\
& \text { Steve:physician [name } \rightarrow \text { “Taylor, Steve“, ..., patients } \rightarrow>\{\text { Bob, Fred, Mary\}] }
\end{aligned}
$$

where ":" is the is-instance-of operator. Alternatively, we use "." expressions like bob.responsible-doctor.degree [d] for bob[responsible-doctor $\rightarrow D$ [degree $\rightarrow d]$ ].

Queries and rules are expressed with the ?- and $\leftarrow$ operator, e.g. (in compact "." expression notation):

?- P:patient $\wedge$ P.diagnosis["lymphoma”] $\wedge$ P.responsible-doctor.name["Taylor, Steve"] // find patients with lymphoma where responsible doctor is Taylor, Steve

D.name["Miller, John"] $\leftarrow P$ :patient $\wedge$ P.diagnosis["leukemia type AML”] $\wedge$ P.responsible-doctor [D]

// if patient suffers from special leukemia of type AML, then Miller, John is the

// doctor of this patient (as, for example, he is the AML-specialist in the hospital)

From $T L$, we use the standard update operators del and ins, which can be applied to every database predicate, e.g. patients.ins (patient-oid) (with patients being the predicate extension of the patient class above). del and ins have a formal semantics (in contrary, for example, to Prolog's assert and retract), and any sequence involving database updates can be specified as a transaction via the serial conjunction operator $\otimes$. This means if any step of the $\otimes-$ sequence fails, the sequence is rolled back.

\footnotetext{
${ }^{1}$ Note that we do not use $T L$ to model the workflows themselves (as, for example, in [DKR+98]).
} 


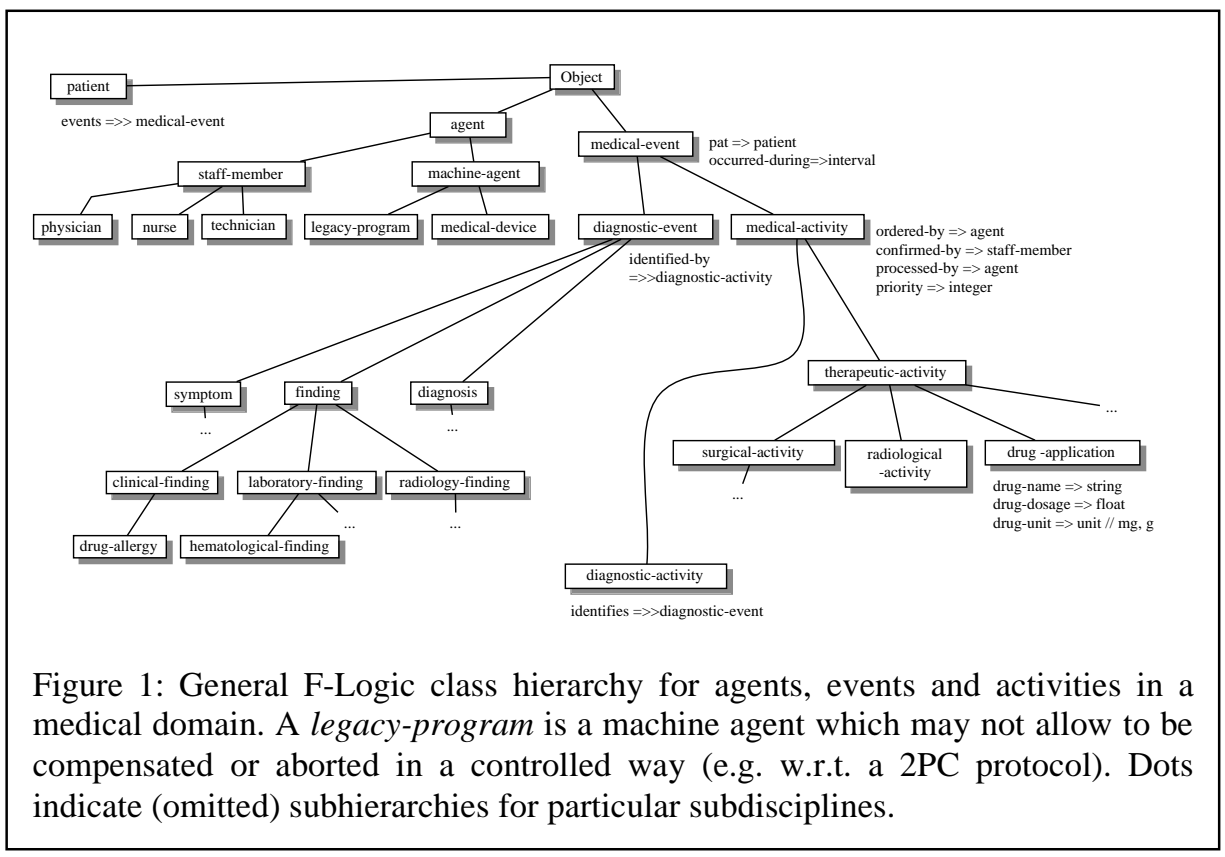

Further $T L$-operators such as the | operator for concurrent execution are not needed here as we do not use $T L$ for workflow definition.

\section{Medical Events and Workflows}

In the following, we formalize our notation of medical events, activities, semantic exceptions, and workflows over medical activities.

\subsection{Medical Events, Activities, and Semantic Exceptions}

Fig. 1 shows the F-Logic data model hierarchy of event and activity classes we use in this paper. The class medical-event is the abstract super class of everything that "happens" to a patient and that has a medical relevance. The medical-event subclass diagnostic-event is the root class for all data describing diagnostic information about the patient, while the subtree starting from medical-activity provides classes for the storage for all medical activities done with the patient (e.g. drug applications). For the purposes of this paper, we assume in a patient database $D B$ the two associated basic predicates:

diagnostic-events $(D)$ with $D$ : diagnostic-event 


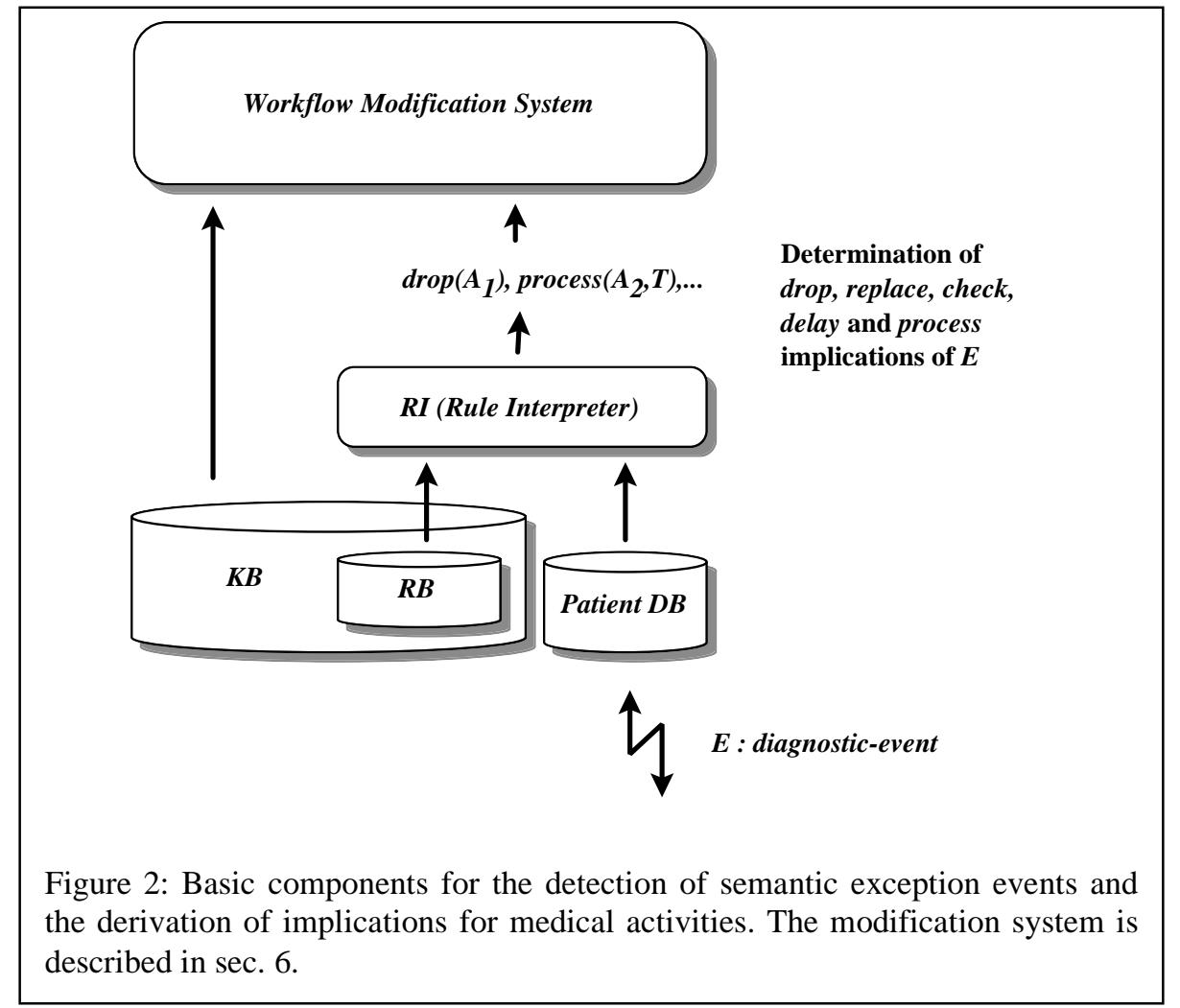

which is the object extension storing all diagnostic events that have been observed or detected w.r.t. the patients, and

\section{activities-ordered (A) with $A$ : medical-activity}

which records all activities that already have been ordered or suggested by human or automated agents (but may not yet have been carried out).

A semantic exception is a diagnostic-event instance which implies that the current treatment has to be modified (e.g. because of an allergy). The interpretation whether or not an event constitutes a semantic exception is the task of a rule base $R B$ and a rule interpreter $R I$. Furthermore, we assume a knowledge base $K B$ incorporating $R B$ and containing patient-independent information about medical events, such as the duration and dosage of a specific drug application (see fig. 2).

\subsection{Derived Predicates on Activities}

As $R I$ derives statements about medical activities, we now introduce predicates on medical-activity instances. Therefore, let Pred $=\{$ drop, replace, check, delay, process $\}$ be our set of activity predicate symbols, $A$ an object of class medical-activity, 


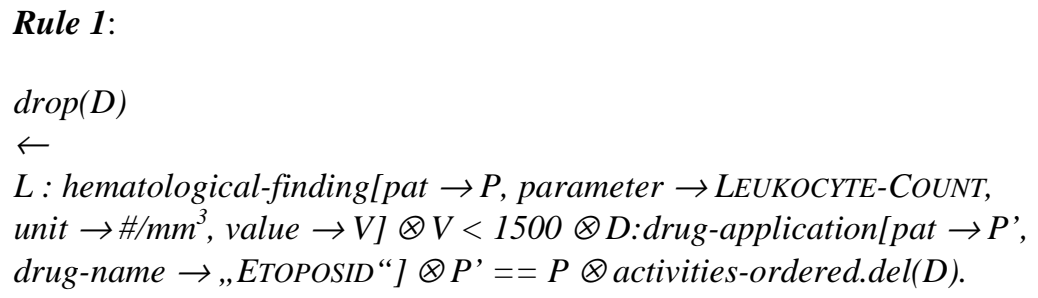

Rule 1:

$\operatorname{drop}(D)$

$\leftarrow$

$L:$ hematological-finding[pat $\rightarrow P$, parameter $\rightarrow$ LEUKOCYTE-COUNT,

unit $\rightarrow \# / \mathrm{mm}^{3}$, value $\left.\rightarrow V\right] \otimes V<1500 \otimes D:$ drug-application [pat $\rightarrow P^{\prime}$,

drug-name $\rightarrow$,ETOPOSID “] $] \otimes P^{\prime}==P \otimes$ activities-ordered.del $(D)$.

Rule 2:

$\operatorname{process}(D,[0,2$ days] $) / /$ give drug $D$ within two days

$\leftarrow$

L:hematological-finding[pat $\rightarrow P$, parameter $\rightarrow$ LEUKOCYTE-COUNT,

unit $\rightarrow \# / \mathrm{mm}^{3}$, value $\left.\rightarrow V\right] \otimes V<2000 \otimes D$ :drug-application $\otimes$ new-id $(D) \otimes$ D.init[pat $\rightarrow P$, drug-name $\rightarrow$,G-CSF“, drug-dosage $\rightarrow 300$, drug-unit $\rightarrow \mu g$ ] $\otimes$ activities-ordered.ins $(D)$

Figure 3: Sample rules deriving implications for medical activities.

and $T$ a temporal constraint. $T$, for example, may specify the interval during which an activity should be processed. Then we define with

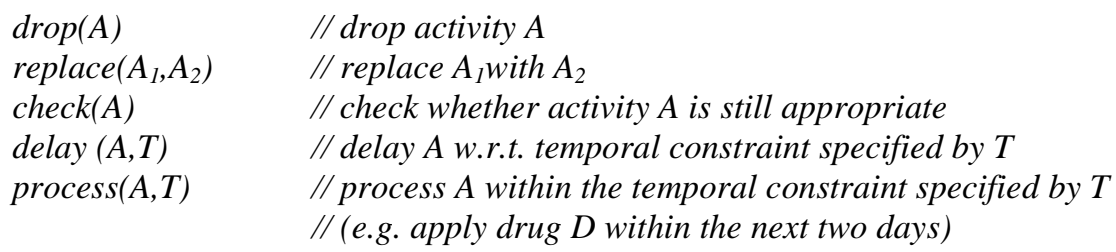

the associated predicate molecules. The first four predicates deal with semantic failures of activities. drop and replace express "hard" failures (with the consequence "do not process activity anymore"), while check and delay address "weak" failures. Fig. 3 contains some sample rules concerning implications of semantic exceptions. Rule 1 orders the discontinuation of the drug ETOPOSID when the leukocyte count is less than 1500 per $\mathrm{mm}^{3}$, while rule 2 suggests the administration of the so-called GRANULOCYTE COLONY STIMULATING FACTOR $(G-C S F)$ for hematological recovery when the leukocyte count is less than 2000.

The main task of the modification system we will describe in sec. 6 is to translate derived predicates on activities into workflow instance modifications. However, we first briefly describe our workflow representation and execution model.

\subsection{Workflow}

In our context, we view a workflow as a control flow over medical activities. A workflow node $n$ represents exactly one activity (e.g. a drug application or a x-ray 
examination) which is processed when the control flow reaches $n .^{2}$ For workflow build-time purposes, we call a construct of the form

$$
\begin{aligned}
& \text { class-name }\left[a_{t t r} \rightarrow^{3} \mathrm{val}_{1}, \ldots, \text { attr }_{n} \rightarrow v \mathrm{vl}_{n}\right] \text { with class-name::medical-activity, } \\
& \text { e.g. } \\
& \text { drug-application[drug-name } \rightarrow \text { “CISPLATIN”,drug-dosage } \rightarrow 200 \text {, drug-unit } \rightarrow m g \text { ] }
\end{aligned}
$$

a medical-activity instance definition. Such an instance definition can be assigned to a node to indicate that the node at run-time manages (i.e. processes) an activity instance with this properties (the definition is called partial if some attribute values are not specified at build-time but will be determined at run-time ${ }^{4}$ ).

Let Activities be a set of (partial) activity instance definitions. A workflow $W$ over Activities is a 6-tuple $W=\left(\right.$ Nodes $\left._{W}, N A M_{W}, \operatorname{Edges}_{W}, \operatorname{ETM}_{W}, E C M_{W}, \operatorname{SemSeq}_{W}\right)$ where

- $\operatorname{Nodes}_{W}$ is a set of workflow nodes with Nodes $_{W}=$ Activity-Nodes $_{W} \cup$ ControlNodes $_{W}$. While Activity-Nodes ${ }_{W}$ is the set of activity nodes, Control-Nodes contains the control flow nodes of types START, END, OR-SPLIT, AND-SPLIT, ORJOIN, LOOP-START, or LOOP-END.

- the function $N A M_{W}:$ Activity-Nodes $\rightarrow$ Activities maps (partial) activity object instance definitions to the activity nodes.

- Edges $_{W} \subset$ Nodes $_{W} \times$ Nodes $_{W}$ is the set of directed (control flow) edges. The subset Edges $_{W, \text { sync }} \subset$ Edges $_{W}$ contains synchronization edges (see, for example, [RD98,ASE+96]). The semantics of a synchronization edge $e=(n, m)$ is that $m$ may only be activated when $n$ has completed, or when $n$ is not reachable anymore by the control flow 5 .

- ETM $_{W}:$ Edges $_{W} \rightarrow$ TemporalObjects assigns a temporal object such as an exact duration (e.g. "4 days") or an interval to an edge to indicate temporal transition conditions such as "Next chemotherapy starts exactly 4 days after previous one". By convention, ETM $M_{W}$ maps the temporal NULL object to an edge when no temporal transition condition exists.

- $E_{C} M_{W}:$ Edges $_{W} \rightarrow$ Conditions assigns a F-Logic condition such as "... [parameter $\rightarrow$ LEUKOCYTE-COUNT, value $\rightarrow V] \wedge V<1500$ " to an edge, which must be fulfilled when the control flow wants to pass an edge. Again, by convention, we set $\operatorname{ECM}_{W}(e)=$ NULL when there is no transition condition.

${ }^{2}$ We could also assign several activities to a node. However, we favor a 1:1 relationship between nodes and activities, as this facilitates and unifies workflow modification such as dropping an activity.

${ }^{3}$ or $\rightarrow>$ for set-valued attributes

${ }_{5}^{4}$ E.g. the particular dosage of a drug may not be determined before node execution.

${ }^{5}$ See [RD98] for a more detailed typology of synchronization edges. For the scope of this paper, however, we restrict these edges to the "standard" type described above. 
- $\operatorname{SemSeq}_{W} \subset\left\{\left\{k_{1}, . ., k_{m}\right\} \subset\right.$ Activity-Nodes ${ }_{W} / k_{1}, . ., k_{m}$ is sequence within $\left.W\right\}$ is the set of activity sequences that should not be broken up by inserting additional activity nodes.

Remarks: The partition of transition conditions into temporal and "non-temporal" conditions is mainly motivated by the introduction of so-called interval covering workflow regions in sec. 5, which need $E M_{W}$ for determination. The problem that a "non-temporal" transition condition such as "LEUKOCYTE-COUNT < 1500" implicitly has a temporal aspect in the meaning of "Wait the number of days until LEUKOCYTECOUNT $<1500$ " is discussed in this sec. too.

$\mathrm{SemSeq}_{W}$ denotes meta information for the modification system w.r.t. the question which sequences - from the application view - form semantic and "atomic" units (e.g. Perform Chemotherapy Toxicity Test $\rightarrow$ Prepare Patient for Chemotherapy $\rightarrow$ Perform Chemotherapy) and should not be broken up by inserting additional activities. In this case, the modification agent, for example, tries to insert a parallel branch for the additional activity. $s \in \operatorname{SemSeq}_{W}$ can be user-defined or constructed by the inter-activity-dependency predicate (see sections 5 and 6).

Activities $_{W}$ denotes the set of all medical-activity instance definitions used by $W$. Furthermore, to reduce formal complexity, we do not consider nested workflow definitions (i.e. workflows with abstract aggregated nodes representing subworkflows). If nested workflows are defined at build-time, we assume that such a workflow definition is transformed at run-time into a flat workflow by replacing the aggregation nodes by their subworkflows.

\section{Workflow Execution and Interruption}

We now formalize our execution model and notion of an event-caused instance interruption: With $i_{W}$, we denote a run-time instance of the definition $W$, and assign a control agent $C A_{i_{W}}$ to every $i_{W}$, which controls and processes the instance. Furthermore, $C A_{i_{W}}$ also is responsible for dynamically modifying the control flow of $i_{W}$ (what will be described in sec. 6). To every instance $i_{W}$, we can assign exactly one object $P$ : patient treated by $i_{W}$. This assignment is expressed by the function getpatient $\left(i_{W}\right)$.

When $i_{W}$ is generated by the engine, the following initializing routine is processed:

for $a \in$ Activities $_{W}: / /$ a is used by $W$

A:medical-activity $\otimes n e w$-id $(A) \otimes \operatorname{copy-values}(A, a) \otimes A$.pat $\left[\right.$ get-patient $\left.\left(i_{W}\right)\right] \otimes$ activities-ordered.ins(A)

where new-id is a $T L$ predicate binding $A$ to a new oid each time this predicate is evaluated $^{6}$, and copy-values a function that copies the values of the partial instance definition $a$ to the attributes of $A$. This is necessary as the automated modification

\footnotetext{
${ }^{6}$ see [KLW95], sec. 17.4, for details concerning object generation in F-Logic/TL
} 
system of sec. 6 has to reason about the activities the instance is currently processing or will perhaps process depending on the control flow. ${ }^{7}$

At the occurrence of an event $E$ during execution, an activity node $n \in$ ActivityNodes has a state described by the function $\operatorname{state}_{E}(n) \in\{$ untouched, activated, currently-processed, committed, failed\}, where

- $\quad$ untouched means that the control flow has not yet reached node $n$ (or will never reach $n$ at all w.r.t. the particular workflow instance $i_{W}$ ).

- activated means that control flow has reached the input edges of $n$, and that the conditions $\operatorname{ETM}(e)$ and $\operatorname{ECM}(e)$ are fulfilled for every input edge $e$.

- currently-processed means that $n$ is currently processed by $C A_{i_{W}}$.

- committed means that the processing of $n$ has been successfully completed.

- failed means that $n$ is not appropriate anymore. Each of the states untouched, activated, currently-processed may change directly to failed. The relationship of the failed state with the failure predicates drop, replace, check and delay of sec. 4.2 is that after $R I$ has derived, for example, a $\operatorname{drop}(A)$ statement for an activity $A$, it is automatically explored which workflow nodes w.r.t. which workflow instances are affected. These nodes are then temporarily set to state failed, and then replaced or dropped by the dynamic modification system (see sec. 6). Technical failures of steps (e.g. because of an engine crash) are beyond the scope of this paper.

\subsection{Exception-Caused Workflow Interruption}

We say that $i_{W}$ has been $E$-interrupted at node set $N_{E}=\left\{n_{1}, ., n_{k}\right\}$, if $C A_{i_{W}}$ has interrupted the control flow of $i_{W}$ because of the occurrence of an event $E$, and if - at the moment of the interruption $-\operatorname{state}_{E}\left(n_{i}\right)$ is activated or currently-processed for all $n_{i}$. The cardinality of $N_{E}$ is $>1$, if $i_{W}$ is interrupted within parallel execution (i.e. after an AND-SPLIT).

\subsection{Exception-Related Workflow Modification Regions}

An important subproblem of dynamic workflow modification is the question for which workflow region it is "sensible" at all to reason about structural changes when a semantic exception occurs. For example, if it is detected during a chemotherapy that a patient has an allergy w.r.t. one of the drugs, it makes sense to delete every node in the workflow instance which applies this drug (as it is too dangerous to administer this drug furthermore). However, if the patient only shows a moderate toxicity w.r.t. an important drug, it makes sense to delete the drug nodes only in a ,nearer“ neighborhood, and to assume that in workflow areas ,far away“ the drug will be applied again, as patients usually recover from moderate toxicity effects and are then able to take this drug again after a while. Furthermore, in domains where semantic

${ }^{7} \mathrm{We}$ omit techniques to iteratively and on-demand generate the medical-activity objects during instance execution, as this is a performance aspect. 
exceptions occur frequently, it is useless to reason about workflow modifications which would affect regions which are - w.r.t. the number of activities - „further away" from the current control flow, as another exception may happen in the meantime creating a new situation. The basic problem of modification regions is, of course, that they hardly can be specified at build time, but depend on the type of the exception event $E$.

For our purposes, we formalize a modification region $(M R)$ as follows: Given a workflow $W$, an event $E$ and an interruption node set $N_{E}=\left\{n_{l}, . ., n_{k}\right\} \subset$ Nodes $_{W}$, a $M R$ $r_{W, E, N_{E}}$ syntactically is a complete subgraph of $W$ containing $N_{E}$ and having the strictforward characteristic. The latter means, that for all $m \in r_{W, E, N_{E}}$, there exists a $n_{m} \in$ $N_{E}$ and a path $n_{m} \rightarrow k_{l} \rightarrow, \ldots, \rightarrow k_{j} \rightarrow m$ with nodes from $r_{W, E, N_{E}}$ (i.e. the control flow starting at $n_{m}$ may reach $m$ during execution) $)^{8}$.

The determination of a $M R r_{W, E, N_{E}}$ is done in three basic steps: First the interval $T$ expressing the "temporal influence" of $E$ is determined. Second, for every path starting from $N_{E}$ a subpath covering $T$ is determined. Third, $r_{W, E, N_{E}}$ is constructed by the union of this subpaths. These steps are now described in detail:

Temporal influence of events: For this, we have to assume some heuristic knowledge about the „temporal scope“ of events. We therefore assume in our medical knowledge base $K B$ patient-independent information about events (similar to the average duration of medical activities above), such as the definition of drug-caused hematological toxicity and the average time a patient needs to overcome such a drug-caused side effect (although this is complex knowledge, it is often already available in current medical knowledge-based systems; see, for example, [MSN+97, MTD+96]). The $K B$ predicate for $M R$ determination is kb-temporal-scope $(k b-E, T)$, where $k b-E$ is a (partial) instance definition of a $K B$ class hierarchy, and $T$ an interval information. $k b$ temporal-scope represents the usual or average time period during which an event with the characteristics of $k b-E$ affects the activities processed for an usual patient. For example, for a drug allergy we would have kb-temporal-scope (kb-drug-allergy[], $[0, \infty])$ to represent that a detected drug allergy influences the therapy of the patient for the whole remaining treatment (whatever its particular characteristics are). In opposite to this, for a moderate hematological side-effect such as a decreased leukocyte count as a consequence of aggressive drugs, we would have

$$
\begin{aligned}
& \text { kb-temporal-scope } \\
& \qquad \begin{array}{l}
\text { kb-hematological-finding } \\
{[\text { type } \rightarrow \text { CYTOSTATIC-DRUG-CAUSED-HEMATO-TOXICITY, }} \\
\text { leukocyte-interval } \rightarrow\left[500 \# / \mathrm{mm}^{3}, 2500 \# / \mathrm{mm}^{3}\right], \\
[0,2 \text { weeks }])
\end{array}
\end{aligned}
$$

\footnotetext{
${ }^{8}$ Non-strict forward-oriented MRs make sense, when we have to consider that the workflow is rolled back and reset to a node from which additional nodes become reachable. The construction of such roll-back insensitive $M R s$ may be appropriate for dangerous events (such as a serious hematological toxicity w.r.t. a drug $D$ ) so that in case of a roll-back it is guaranteed that $D$ will also not be applied. Because of limited space, we do not address this type of MRs.
} 
to represent that such an event influences the workflows only for a period of about 2 weeks (during which the responsible drugs should be dropped), as it can be assumed that the patient will recover during these two weeks and will be able to take the drugs again ${ }^{9}$.

Interval covering region: Given a workflow definition $W$, a node $n$ and an interval $T$ of type [0, $k$ time-units], the $T$-covering region $r_{\text {cover, } W, n, T}$ is a strict forward-oriented region that covers those nodes that will - in the normal case (i.e. if no additional semantic or technical exceptions occur) - be processed during the interval $T$ (beginning with $n$ ). The determination of a $T$-covering region $r_{\text {cover, }, n, T}$ is achieved as follows: For every path $p_{n}$ starting from $n$ the following is done: for the direct successor activity node $m$ of $n$ (w.r.t. $p_{n}$ ) heuristic information in the knowledge base $K B$ about the normal duration of the associated activity is retrieved. Then, the transition time w.r.t. the successor of $m$ is determined (by using the function $E T M_{W}$ ). This is done iteratively until the subpath $p^{\prime}{ }_{n} \subset p_{n}$ is found with the characteristic that the subpath $\left(p^{\prime}{ }_{n}+\right.$ next successor) would exceed the duration specified by $T . r_{\text {cover, }, n, T}$ is then constructed by the union of all of these subpaths $p^{\prime}{ }_{n}$ originating from $n$.

Remark: The determination of $r_{\text {cover, } W, n, T}$ faces two problems: First, if for an edge $e$ of a path $p_{n}$ it holds that $E C M_{W} !=N U L L$, this means that the transition duration is unpredictable at determination time of $r_{\text {cover, } W, n, T}$. At the moment, we consider such an $e$ simply with a duration of $\max (0, \operatorname{ETM}(e))$, with the consequence that $r_{c o v e r, W, n, T}$ may contain nodes that are executed after the interval $T$. In other words, the nodes of $r_{\text {cover, } W, n, T}$ in this case form a superset of the nodes executed during $T .{ }^{10}$ Second, if a $p_{n}$ contains a loop with a termination condition such as Do Chemotherapy activities until $x$-ray cannot detect any tumor remainder anymore the execution time of the loop is unpredictable too. Again, without additional meta knowledge we can only assign a default duration such as the duration of its activity sequence to this loop, with the consequence that $r_{\text {cover, } W, n, T}$ again becomes a non-minimal $T$-covering region. In both cases, $r_{\text {cover }, W, n, T}$ only is "close" to the region which will in fact be executed during $T$. However, this is still better than changing the workflow w.r.t. regions which are not relevant at all.

Final construction: The $M R r_{W, E, N_{E}}$ is then constructed by the union of all $r_{c o v e r, W, n, T}$ for $n \in N_{E}$.

\footnotetext{
${ }^{9}$ If not, the rule system would derive another semantic exceptions after this period stating that the patient shows an exceptional recovery pattern that requires additional procedures.

${ }^{10}$ The determination of the minimal $r_{\text {cover, } W, n, T}$ in such cases would require additional meta knowledge such as the average time $t_{C \text {, lastValue }}$ it takes for a patient after a chemotherapy of type $C$ to increase the LEUKOCYTE-COUNT from lastValue to 1500. As there exist detailed biomathematical models for cell colony growing in the oncological domain, we can assume this knowledge, for example, for HEMATOWORK.
} 


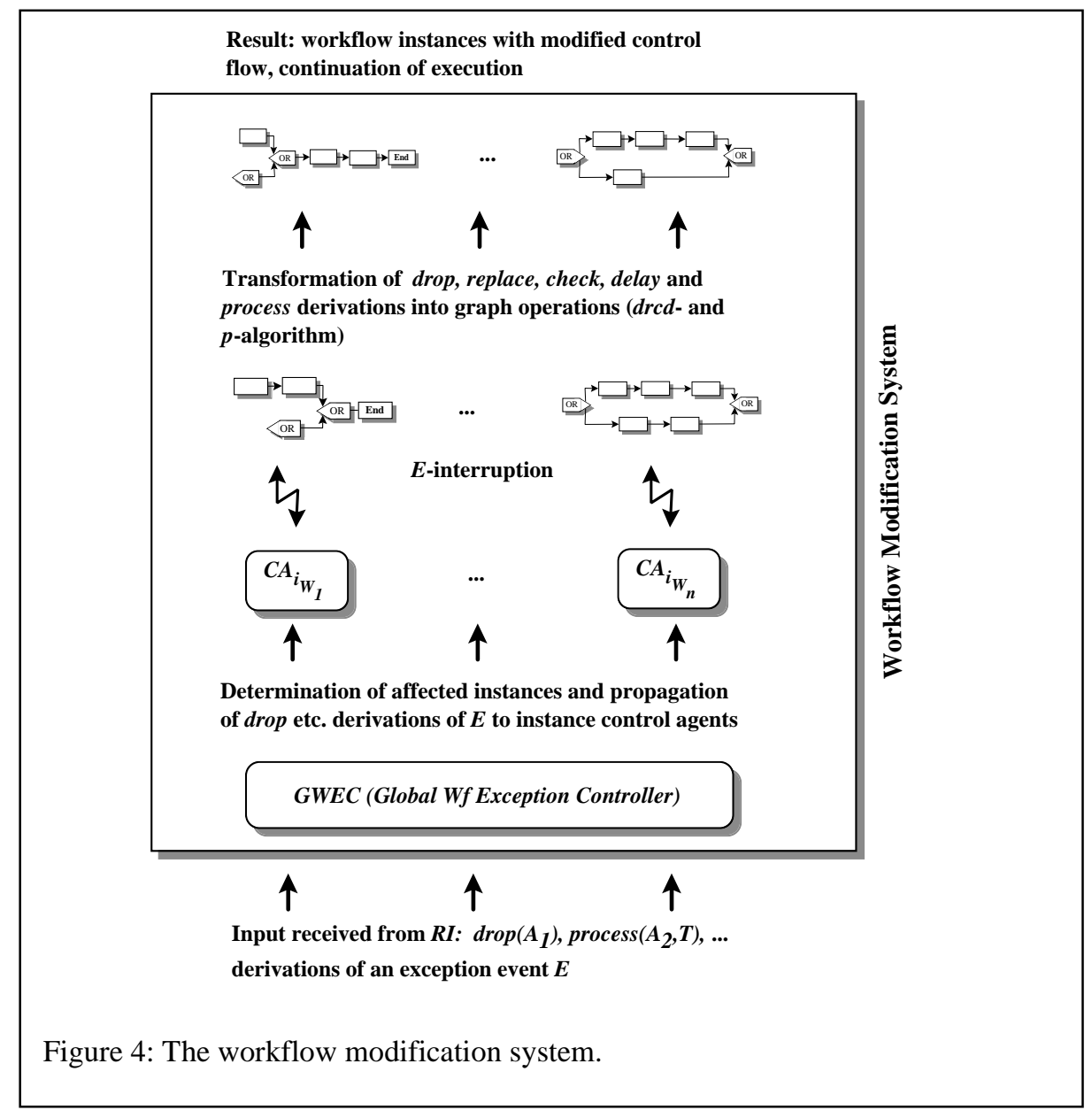

\section{The Dynamic Workflow Modification System}

We now describe the dynamic workflow modification system in detail (see fig. 4 for an overview). We therefore assume that $E$ is a new event inserted into diagnosticevents $(D)$ in the patient $D B$ (of fig. 2). This triggers the rule interpreter $R I$, which fires a set of rules $R_{I}, \ldots, R_{n}$. For the sample rules of fig. 3, the event

$$
\text { E : hematological-finding[ parameter } \rightarrow \text { LEUKOCYTE-COUNT,..,value } \rightarrow 1200]
$$

would induce rules 1 and 2, which would derive that the drug ETOPOSID should be dropped and that for hematological recovery an additional drug, the so-called G-CSF (GRANULOCYTE COLONY STIMULATING FACTOR), should be given. 
$R I$ then passes the set of all derived predicates $d p=\left\{\operatorname{drop}\left(A_{1}\right), \ldots, \operatorname{replace}\left(A_{m}\right.\right.$, $\left.\left.A_{m+1}\right), \ldots, \operatorname{delay}\left(A_{n}, ..\right), \ldots, \operatorname{check}\left(A_{o}\right), \ldots, \operatorname{process}\left(A_{p}, ..\right), \ldots\right\}^{11}$ to the global workflow exception controller GWEC. This agent, which constitutes the first layer of the modification system, identifies the set of possibly affected instances pai $i_{E}=\left\{i_{W_{l}}, \ldots, i_{W_{n}}\right\}$ for the respective patient (by checking whether get-patient $\left(i_{W}\right)=$ E.pat holds). The further modification steps depend on the activity predicate type and are described in sections 6.1 and 6.2 .

An important subproblem w.r.t. all predicate types is to determine whether a modified workflow definition leads to new inter-activity dependencies which have to be expressed. For example, if it is necessary to insert a new activity node, it must be decided whether this node can be directly executed ,after“ the interruption location, or whether inter-activity constraints do require that it has to be executed somewhere later on. For instance, if it is has been derived that a drug $D_{l}$ should be given additionally, but that $D_{1}$ should never be applied directly before a drug $D_{2}$ (as $D_{2}$ may neutralize or weaken the effects of $D_{l}$ ), then the node representing the application of $D_{1}$ must be executed after the $D_{2}$ node.

To handle this problem of inter-activity constraints, we assume in our knowledge base $K B$ information about dependencies w.r.t. the temporal order or the exclusion of medical activities. In particular, we assume a predicate of the form

inter-activity-dependency $\left(A_{1}, A_{2}, T y p e, T\right)$

where $A_{i}$ is a medical-activity instance definition (see sec. 4.3). Type may, for example, have the values BEFORE or EXCLUSION, and $T$ is an (optional) temporal object such as an interval. For example, the predicate

$$
\begin{aligned}
& \text { inter-activity-dependency } \text { drug-application[drug-name } \rightarrow \text { “VINDESIN"], } \\
& \text { drug-application[drug-name } \rightarrow \text { "CISPLATIN"], } \\
&\text { BEFORE, } 1 \text { hour })
\end{aligned}
$$

expresses that the drug VINDESIN must be given exactly one hour before the drug CISPLATIN. This knowledge type is already incorporated in many medical knowledgebased systems such as the one of HEMATOWORK.

\subsection{Management of drop, replace, check and delay derivations (drcd-algorithm)}

To identify the set of in fact affected instances $a i_{E}$, drop, replace, check, delay, it is first checked by $G W E C$ for every $i_{W} \in$ pai $_{E}$, whether an activity $A_{k}$, for which drop, replace, check or delay has been derived, matches at least one entry of Activities ${ }_{W}$.

For $i_{W} \in a i_{E, \text { drop, replace, check, delay }}, G W E C$ then sends an interrupt request to $C A_{i_{W}}$, which performs an $E$-interrupt of $i_{W}$ at a node set $N_{E}$. $C A_{i_{W}}$ now determines the $E$ -

${ }^{11}$ We assume that the knowledge base itself has checked that its knowledge is consistent in the meaning that, given any event constellation, it cannot be derived that an action $A$ has to be processed and has to be aborted simultaneously. This can achieved by the usual knowledge verification strategies (e.g. [SSS82]). 
related $M R r_{W, E, N_{E}}$ and - within $r_{W, E, N_{E}}$ - the set of affected activity nodes by using the function $N A M_{W}$. In the example of fig. 5, where the large rectangle represents the $M R$ for the considered event $E$, it would be detected that the derived predicate of rule 1 in fig. 3 ("drop drug with name ETOPOSID") affects node $l_{l}$, as its activity instance definition represents the application of this drug. Depending on the particular predicate type (drop, replace etc.), graph operations are then started to, for example, eleminate nodes from the control flow (as the node $l_{l}$ in fig. 5). In the case of a replace predicate (new node!) or delay predicate (existing node at new location!), $C A_{i_{W}}$ furthermore inspects the inter-activity-dependency $\left(A_{1}, A_{2}, T y p e, T\right)$ predicate in $k b$ to check if there are dependencies with other existing activities. A BEFORE dependency, for example, is translated into a synchronization edge which is inserted into the workflow graph as well.

\subsection{Management of process derivations (p-algorithm)}

The insertion of new nodes (because of a derived process $\left(A_{\text {new }}, T\right)$ predicate) is different from the dropping or replacing of nodes, as we first have to identify where the node should be placed at all.

The brute method simply could be to generate a new workflow instance with the new activity as the only node. However, this strategy is not appropriate in scenarios where semantic exceptions occur frequently (such as in HEMATOWORK). First, workflow management overhead would be a consequence, because the system must initialize, register etc. a lot of small instances. Second, dependencies between the new activity and activities of already running instances would be difficult to manage.

If we therefore want to insert the new activity into existing instances, we have to decide which instance $i_{W}$ with get-patient $\left(i_{W}\right)=$ E.pat should take over the task of performing this new activity. This problem certainly cannot be solved in general, as it depends on the particular structure of the workflow system and application domain. However, we can identify the following possibilities of a semi-automated identification of an appropriate $i_{W}$ :

- Identification by meta knowledge: This means that GWEC can access basic meta information about the semantic scope of an $i_{W}$. For example, in HEMATOWORK, for every treated patient there are two workflow instances, one for the therapeutic activities and one for monitoring tasks for the parallel diagnostic control. Therefore, if the derived activity would be of type therapeutic-activity, then HEMATOWORK's GWEC would select the therapeutic instance.

- Using Activities $_{W}$ : If the provided high-level meta knowledge is not sufficient, we can directly use the set Activities $_{W}$, which contains the activity instance definitions referenced by $W$ (sec. 4.3). This set, however, may contain an arbitrary mixture of diagnostic and therapeutic activities. Therefore, we can only use aggregation strategies such as determining the cardinality of the subset of diagnostic or therapeutic activities, to identify if $W$ is a "more" diagnostic or therapeutic workflow, and to derive some statements about the semantic scope of $W$. 


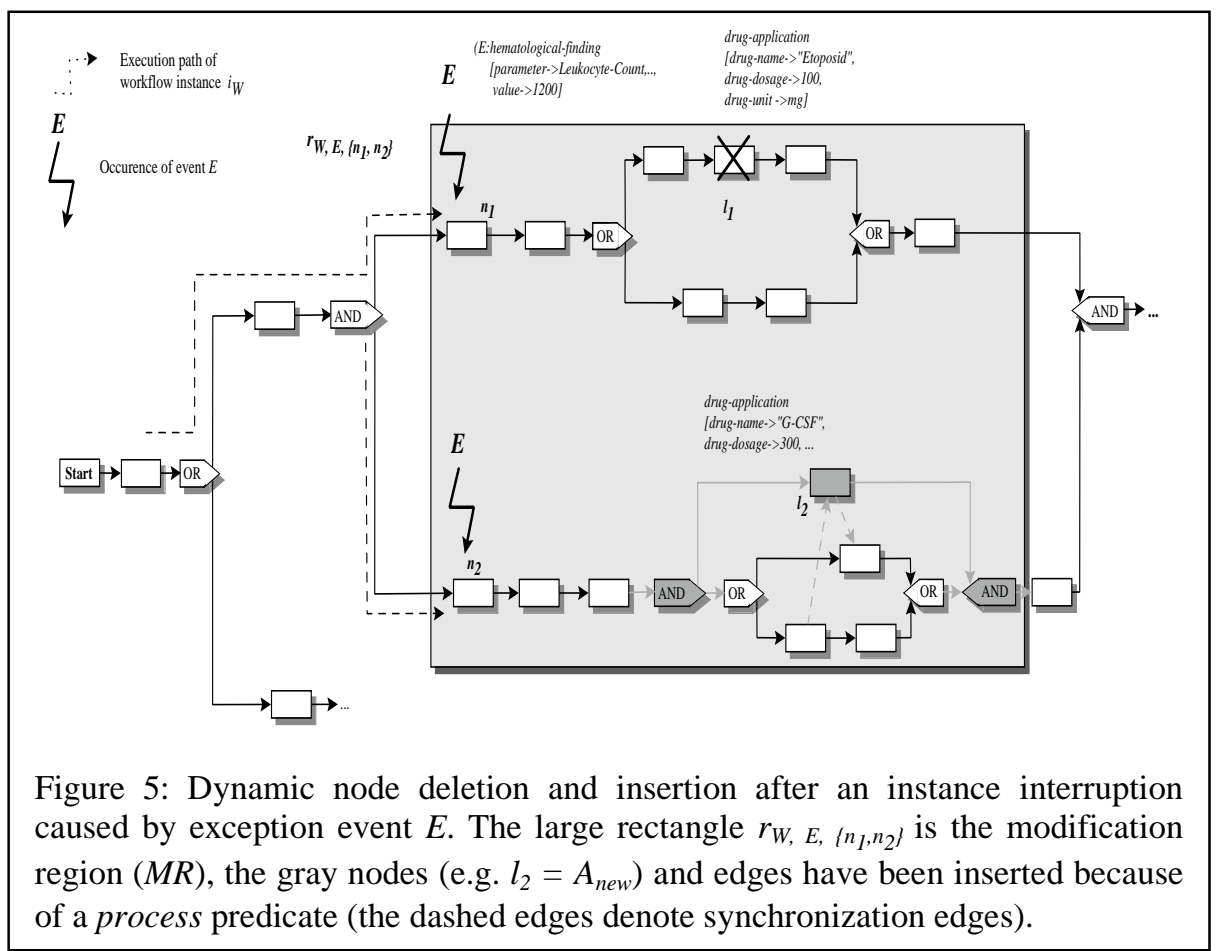

- User-driven selection: If an automatic identification is not possible, the domain expert has to select the appropriate $i_{W}$. This can be used in combination with the two strategies described before (e.g. automated suggestion, confirmation by user).

If an $i_{W}$ has been identified for the new activity $A_{\text {new }}$ and then $E$-interrupted, it has to be decided how the $A_{\text {new }}$-node has to be inserted.

Principally, there are two ways to integrate $A_{\text {new }}$ into $M R$ : It can be inserted into an existing sequence (1), or we can add an AND-SPLIT/AND-JOIN-sphere, which contains (only) the $A_{n e w}$-node as one parallel branch, and the already existing "local" nodes as the other parallel branch (2). Possibility (1), for example, is not appropriate, when a sequence $A_{l} \rightarrow \ldots \rightarrow A_{n}$ being a candidate for insertion of $A_{\text {new }}$ forms a logical unit (i.e. is an element in $\operatorname{SemSeq}_{W}$ ) that should not be "destroyed" by inserting other activities. If the sequence $A_{l} \rightarrow \ldots \rightarrow A_{n}$ allows the integration of $A_{\text {new }}$, we have to consider ordering constraints stored in the inter-activity-dependency predicate, and perhaps have to reorganize the sequence. Otherwise, if an AND-sphere is inserted, ordering constraints have to be managed via the additional insertion of synchronization edges. In particular, the $p$-algorithm ( $p a$ ) works as follows: (see [Mül99] for the full algebraic notation): 
After the $E$-interrupt of $i_{W}$ at node set $N_{E}$, pa first selects a $n \in N_{E}$ as starting point $^{12}$. It then determines the relevant modification region $M R$ which is the intersection of the $E$-related $M R r_{W, E, N_{E}}$ and the $T$-covering region $r_{\text {cover, }, n, T}$. Within $M R$, pa determines the sequence $n=k_{1}, \ldots, k_{m}=c$, where $c$ is the next control node of type AND-SPLIT/OR-SPLIT (or the last node of the sequence within $M R$ ). If $k_{2} \rightarrow, \ldots, \rightarrow$ $k_{m-1}$ is not an element in $\operatorname{SemSeq}_{W}, A_{n e w}$ is inserted into this sequence in a way not violating any constraints in inter-activity-dependency(...). If $\left(k_{2} \rightarrow, \ldots, \rightarrow k_{m-1}\right) \in$ SemSeq ${ }_{W}$, or no constraint-compliant sequence $k_{2} \rightarrow, \ldots, A_{\text {new }}, \ldots, \rightarrow k_{m-1}$ is possible, $p a$ alternatively inserts an AND-SPLIT after $n$ and an AND-JOIN before $c$, and inserts ANDSPLIT $\rightarrow A_{\text {new }} \rightarrow$ AND-JOIN as one branch, and AND-SPLIT $\rightarrow k_{2} \rightarrow, \ldots, \rightarrow k_{m-1} \rightarrow$ AND-JOIN as the other path. However, if an inter-activity dependency of type EXCLUSION with one $k_{i}$ does not allow the insertion of $A_{\text {new }}$ in the $\left(k_{2} \rightarrow, \ldots, \rightarrow k_{m-1}\right)$ context at all, $p a$ moves to the $c$-node. If $c$ is an AND-SPLIT, $p a$ tries to insert $A_{\text {new }}$ into one of the parallel sequences after $c$ by the same criteria described above. In case of $c$ is an OR-SPLIT, $p a$ tries to directly insert an AND-SPLIT before $c$ and an ANDJOIN after the corresponding OR-JOIN, with AND-SPLIT $\rightarrow A_{\text {new }} \rightarrow$ AND-JOIN as one parallel path and the OR-sphere as the other parallel path (see fig. 5). This moving to the next SPLIT-control is done iteratively until $A_{\text {new }}$ has been inserted, or until $p a$ leaves $M R$. In the latter case, additional user input is required specifying, for example, that a sequence in $M R$ is not anymore in $\operatorname{SemSeq}_{W}$, and therefore can be used for the insertion of $A_{\text {new }}$.

After $A_{n e w}$ has been successfully inserted, the next step now is to determine interactivity dependencies w.r.t. already existing activities. This is done by scanning interactivity-dependency $(A, B, T y p e, T)$ in $k b$, and translating them, in the case of a BEFORE dependency, into a synchronization edge. In fig. 5 , for example, $A_{\text {new }}=l_{2}$ (a G-CSFnode) is synchronized with two other nodes.

\section{Summary, Discussion and Future Work}

We described an approach for automated detection of semantic exceptions, and the partially automated derivation of the implications for running workflow instances. The approach is mainly motivated by our experiences with the system HEMATOWORK supporting long-term treatment in cancer therapy. One basic problem of domains such as oncology is the enormous amount of diagnostic and monitoring data, and that a significant number of patients suffer from very specific drug-side effects and other exceptions, so that their treatment workflows have to be modified partially. Therefore, the approach basically intends first to filter exception events out from "normal" events by a rule base, second to automatically derive which workflow instances are affected w.r.t. which control flow areas, and third to automatically adjust - as much as possible - the affected areas w.r.t. their control flow (by dropping and adding new activities). For this purpose, two basic control flow modification algorithms ( $d r c d$-and $p$-algorithm) have been introduced. Even if they fail (if, for example, the $p$-algorithm

\footnotetext{
${ }^{12}$ Please recall that the cardinality of $N_{E}$ is $>1$ iff the interruption occurred during a parallel path execution. Therefore, $A_{\text {new }}$ must be integrated only into one sequence.
} 
is not able to determine an insertion point meeting all constraints), and modification control must be shifted to the domain expert, the system can provide at least information in which region the insertion should take place.

Of course, argument could be made, that the cascade of deletion and insertion operations may lead to incorrect workflows. The counter-argument is, that as long as the original workflow definitions and the underlying rule base are consistent, the modified workflow should be consistent as well. Consistency of rule bases has been addressed by several artificial intelligence approaches (e.g. [SSS82]), while semantic consistency of (human-defined) workflows still is an open research problem, which is, however, orthogonal to our approach of translating rules as procedural fragments into workflow modification operations.

Furthermore, the modification agents described need a lot of declarative knowledge to derive modification implications when events occur. While this may not be readily available in all domains, this approach is well suited for medical domains where knowledge bases are commonly available and can thus be extended for the additional purposes. A further characteristic of our approach is the high percentage of temporal aspects, as we, for example, have to determine appropriate modification and interval-covering workflow regions. Currently, we use user-defined "temporal" objects to express heuristic temporal constraints, but a more explicit system-supported approach by using temporal databases in connection with temporal logic could, from the formal point of view, model some aspects better.

At the moment, we are only able to delete or insert single nodes according to derived predicates which definitely could have been evaluated to true. However, as changing a workflow instance must be viewed as a very time-consuming process, it is desirable to support larger workflow changes. For this, it can be useful to insert larger subgraphs containing activity nodes which may not necessarily will be processed but are likely - because of the particular type of $E$ - to become relevant during further execution of the workflow instance $i_{W}$. For example, if it has been derived that a drug $D$ should be applied because of an event, and if it is known that most of the patients receiving this drug have some side-effects after a few days implying additional measures, it makes sense to directly integrate this additional activities into the control flow, as it is likely that the patient will need at least one of these additional activities. Further work will concentrate on these topics.

Furthermore, the implementation of HEMATOWORK is still one of the major efforts to be addressed. Currently, we have developed the patient database $D B$ based on ORACLE and a knowledge base $K B$ realized with $\mathrm{O}_{2}$. Rules and rule processing modules $(R I)$ are implemented with the rule shell CLIPS (C-LANGUAGE INTEGRATED PRODUCTION SYSTEMS; [GR93] $)^{13}$. The implementation of the engine still is an open problem, as the commercial workflow systems we inspected do not provide sufficient support w.r.t. run-time interrupts and dynamic changes. Therefore, at the moment, an own implementation currently is addressed, based on the CORBA implementation of IONA ORBIX.

${ }^{13}$ Although there exists an available prototypical implementation of F-Logic (FLORID $[\mathrm{FHK}+97])$, we had to implement our rule-related modules with a low-level rule shell (i.e. CLIPS) because of the restricted API capabilities of FLORID. 


\section{Acknowledgements}

We are grateful to M. Reichert, who has given valuable comments especially w.r.t. the graph modification algorithms. Furthermore, we want to thank the anonymous reviewers for their comprehensive suggestions to improve the paper.

\section{References}

ASE+96 Attie, P.; Singh, M.P.; Emerson, E.A.; Sheth, A.; Rusinkiewicz, M.: Scheduling Workflows by Enforcing Intertask Dependencies. Distributed Systems Engineering Journal, vol 3, 1996: 222-238.

BJ96 Bussler, C.; Jablonski, S.: Die Architektur des modularen WorkflowManagement-Systems MOBILE. In [Vos96]: 369-388.

BK94 Bonner, A.J.; Kifer, M.: An Overview of Transaction Logic. Theoretical Computer Science, 133: 205-265.

BK95 Bonner, A.J.; Kifer, M.: Transaction Logic Programming. Technical Report CSRI-323, Computer Science Research Institute, University of Toronto, 1995.

CGP+97 Casati, F.; Grefen, P.; Pernici, B.; Pozzi, G.; Sanchez, G.: WIDE Workflow Model and Architecture. Technical Report, University of Milano, 1997.

DKÖ+98 Dogac, D.; Kalinichenko, L.; Özsu, T.; Sheth; A. (eds.): Workflow Management Systems and Interoperability. Springer, Berlin, 1998.

DKR+98 Davulcu, H.; Kifer, M.; Ramakrishnan, C.R.; Ramakrishnan, I.V.: Logic-Based Modeling and Analysis of Workflows. Proc. PODS98: 2533.

FHK+97 Frohn, J.; Himmeröder, R.; Kandzia, P.-Th.; Lausen, G.; Schlepphorst, C.: FLORID - Ein Prototyp für F-Logik. Proc. BTW97, Ulm, 1997: 100117.

GR93 Giarratano, J.; Riley, G.: Expert Systems: Principles and Programming. PWS Publishing Company, 1993.

HSS96 Heinl, P.; Schuster, H.; Stein, K.: Behandlung von Ad-hoc-Workflows im MOBILE Workflow-Modell. ITG Fachbericht, STAK '96 Softwaretechnik in Automation und Kommunikation, München, März, 1996.

KLW95 Kifer, M.; Lausen, G.; Wu, J.: Logical Foundations of Object-Oriented and Frame-Based Languages. Journal of the ACM, 42(4), 1995: 741843.

MH98 Müller, R.; Heller, B.: A Petri Net-based Model for Knowledge-based Workflows in Distributed Cancer Therapy. Proc. Intern. EDBT98 Workshop on Workflow Management Systems, Valencia, Spain, March 1998.

MHL+98 Müller, R.; Heller, B.; Löffler, M.; Rahm, E.; Winter, A.: HematoWork: A Knowledge-based Workflow System for Distributed Cancer Therapy. Proc. of the GMDS98, Bremen, Sep. 98.

Mü199 Müller, R.: Rule-based Interruption and Modification of Workflow Instances. Technical Report. Department of Computer Science, Leipzig 
University 1999.

MSN+97 Müller, R.; Sergl, M.; Nauerth U. et al.: TheMPO: A Knowledge-Based System for Therapy Planning in Pediatric Oncology. Computers in Biology and Medicine vol. 27(3), 1997:177-200.

MTD+96 Musen, M.A.; Tu, S.W.; Das, A.K et al.: EON: A Component-Based Architecture for Automation of Protocol-Directed Therapy. Journal of the American Medical Informatics Association 1996 (3): 367-388.

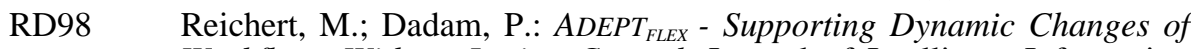
Workflows Without Losing Control. Journal of Intelligent Information Systems 10, 1998: 93-129.

She97 Sheth, A.: From Contemporary Workflow Process Automation to Adaptive and Dynamic Work Activity Coordination and Collaboration. Proc. DEXA Workshop on Workflow 1997.

SK98 Sheth, A.; Kochut, K.: Workflow Applications to Research Agenda: Scalable and Dynamic Work Coordination and Collaboration Systems. In [DKÖ+98]: 35-60:

SSS82 Suwa, M.; Scott, A.C.; Shortliffe, E.H.: An approach to verifying completeness and consistency in a rule-based expert system. AI Magazine 3, 1982:16-21.

Vos96 Vossen, G.: Geschäftsprozeßmodellierung und Workflow-Management: Modelle, Methoden, Werkzeuge. Thomson, Bonn, 1996.

VW98 Vossen, G.; Weske, M.: The WASA Approach to Workflow Management for Scientific Applications. In [DKÖ+98]: 145-164.

Wes98 Weske, M.: Flexible Modeling and Execution of Workflow Activities. Proc. $31^{\text {st }}$ Hawaii Int'l Conf. on System Sciences (HICSS-31), Software Technology Track (Vol VII), 1998: 713-722. 